\title{
Towards a Comprehensive Understanding of Agile Teamwork: A literature-based Thematic Network
}

\author{
Arthur Freire*, Manuel Neto*, Mirko Perkusich*, Alexandre Costa*, \\ Kyller Gorgônio*, Hyggo Almeida*, Angelo Perkusich* \\ * Intelligent Software Engineering (ISE) Group @ VIRTUS, Federal University of Campina Grande \\ Emails: \{arthurfreire, manuel\}@ copin.ufcg.edu.br \\ and \{mirko, alexandre.costa, kyller, hyggo, perkusic\}@ virtus.ufcg.edu.br
}

\begin{abstract}
-
Agile Software Development (ASD) has become the mainstream software development method of choice. Its core fundamentals are based on Teamwork factors and the higher value it gives to individuals and their interactions over processes and tools. Teamwork and human factors have been addressed as essential topics in the literature, and researchers have stated the importance of measuring it to increase the chances of success of ASD projects. However, there is no common understanding regarding the factors that should be considered for defining an ASD Teamwork construct. Driven by this problem, this paper presents a thematic network that defines the themes (i.e., factors) that should be considered when addressing ASD Teamwork. The ASD Teamwork thematic network is the result of a process that consisted of (i) defining the studies used as a data source through a literature review; (ii) extracting data from these studies; (iii) translating this data into codes; (iv) translating the codes into themes; (v) creating the model of higher-order themes; and, (vi) assessing the trustworthiness of the synthesis. The resulting thematic network comprises four higher-level themes: Cohesion, Orientation, Shared Leadership, and Autonomy. We believe that the constructed thematic network can be generalized to ASD and used as the basis by researchers who intend to explore ASD Teamwork. Further, practitioners can use our results to understand agile teams' dynamics better and improve their efficiency.
\end{abstract}

Index Terms-Teamwork; Agile Software Development; Agile; Thematic Network; Construct

\section{INTRODUCTION}

According to Hoda et al. [1], Agile Software Development (ASD) has become the mainstream development method of choice. The main reason for adopting ASD is its capability to respond to environmental changes, such as requirements, quickly. Usually, agile initiatives embrace iterative development, which means dividing the delivery process into short iterations, allowing requirements to be refined on a regular basis [2].

Additionally, to enable responsiveness to change, the Agile Manifesto [3] states that ASD values individuals and interactions more than processes and tools [4]. The team's importance in ASD is evidenced by the Agile Manifesto having six out of the twelve principles directly related to the team (i.e., individuals). Moreover, other researchers have recently assessed the relationship of team members' personality on Teamwork quality (TWQ) in the context of ASD [5].
Research has shown that TWQ has a positive impact on team development [6], [7], and is, consequently, essential for succeeding with ASD [8], [9], [10], [11], [12]. Batista et al. [13] discussed that the effective combination of individual parts, often carried out by software development teams, requires interactions among team members and the coordination of interdependent individual and team level tasks. Given its impact, researchers argued about the importance of assessing TWQ to increase the chances of succeeding with ASD [13], [14].

In this context, researchers have proposed instruments for assessing ASD Teamwork quality. Moe et al. [15] presented a Radar Plot that considers five dimensions for assessing TWQ: Shared Leadership, Orientation, Redundancy, Learning, and Autonomy. Lindsjørn et al. [16] presented a Structural Equation Model, based on a differentiated replication [17] from [6], which considered that the Teamwork construct is comprised of six variables: Communication, Coordination, Balance of Member Contribution, Mutual Support, Effort, and Cohesion. Finally, Freire et al. [14] proposed a Bayesian networksbased model with 16 variables, which had its practical utility positively assessed in a case study.

Unfortunately, there is still no common understanding of what factors should be considered in the ASD Teamwork construct. Except for the instrument proposed by Freire et al. [14], which claimed to have considered the factors presented in Lindsjørn's et al. [16] and Moe's et al. [15] instruments, there is no direct similarity between any of the ASD Teamwork factors in these last two instruments. Even though Freire et al. [14] considered factors from the other two instruments, it does not contain them all; it does not include the variable Balance of Member Contribution, which is part of the ASD Teamwork construct presented in [16]. Moreover, except from the ASD Teamwork factors presented in [14], we did not find other study in the literature that presented a similar list based on the literature. Therefore, we believe that there is still a need to develop a comprehensive and shared understanding of the essential factors included in the ASD Teamwork construct.

To address this gap, we identified the ASD Teamwork factors presented in the literature through thematic analysis, following the guidelines presented by Cruzes and Dyba [18]. This paper presents the employed methodology and the resulting thematic network, which comprises the factors (i.e., 
themes) for ASD Teamwork and their relationships.

The remainder of this paper is organized as follows. Section II presents necessary background and related work. Section III presents the employed methodology. Section IV describes the resulting thematic network. Section V discusses this study's findings in light of its implications for research and practice. Section VI discusses this study's threats to validity. Finally, Section VII presents our final remarks.

\section{BACKGROUND AND RELATED WORKS}

Freire et al. [14] present a Bayesian networks-based model to assess and improve the TWQ in the ASD context. To build the model, the authors listed many ASD Teamwork key factors extracted from the literature. Based on the knowledge of an expert and the resulting list, they used reasoning on a top-down approach - starting with the target node (i.e., Teamwork quality) - breaking down higher-level factors into others they judged more observable. In [14], the authors also present the results of a case study in which their instrument's practical utility was assessed. They concluded that their model helps agile teams assess TWQ and identify improvement opportunities, is easy to learn, and the cost-benefit for using it with the proposed procedure is positive.

Lindsjørn et al. [16] presented a Structural Equation Model instrument, based on a differentiated replication [17] from a study of Hoegl and Gemuenden [6], which considered that the Teamwork construct is comprised of six variables: Communication, Coordination, Balance of Member Contribution, Mutual Support, Effort, and Cohesion. Lindsjørn et al. assumed that study presented in [6] focused on traditional software development methodologies and analyzed how the theory presented in it applies to ASD. As a result, they concluded that the quality of the Teamwork is a major factor in improving team performance, especially for the product's quality.

Moe et al. [15] propose a Radar plot-based instrument to help diagnose agile Teamwork, which considers five dimensions: Shared Leadership, Orientation, Redundancy, Learning, and Autonomy. The instrument was presented to a group of experts comprised of 35 people. They found the model useful for understanding team problems, such as the team agreeing on using test-driven development. According to the authors, the instrument gives researchers and developers a common language for discussing Teamwork.

\section{RESEARCH METHODOLOGY}

This section presents the employed research methodology and partial results - due to space limitations - of the steps executed for constructing the proposed thematic network. This study aimed to understand the factors (or dimensions) of agile Teamwork. Given this, we defined the following Research Question (RQ):

RQ: Which themes should be considered when defining ASD Teamwork?

To answer RQ, we employed a thematic analysis approach [18]. The employed process can be divided into two main steps: (i) Data Source Definition and (ii) Thematic Network Construction. The output of step (i) was used as input for the employed technique we applied in step (ii). In what follows, Section III-A describes step (i) and Section III-B, step (ii).

\section{A. Data Source Definition}

The first step was to define our data source, in other words, identify studies that present ASD Teamwork factors. For this purpose, we used as the initial set of studies the fifteen studies pointed by Freire et al. [14] (i.e., seed set). Later, we employed a Forward Snowballing [19] to identify additional primary studies. To select the studies to be considered on the Forward Snowballing, we employed the following inclusion criteria:

1) Published in 2019 or later

2) Written in English

3) Published in a Conference proceedings or Journal as full papers

4) Focused on industry context (i.e., not considering papers/studies with students or in the academic context)

5) Presents ASD Teamwork factors

We decided to define stringent criteria to consider a paper relevant in the Forward Snowballing step because we assumed our start set to be reliable. Such reasoning follows from it being based on a previews literature review discussed in Freire et al. [14]. Further, our goal was not to quantify the frequency of appearance of a factor in the literature in favor of its relevance, but to identify high-quality studies that discussed relevant agile Teamwork factors.

We managed the Forward Snowballing process in an online Google Spreadsheet (see Appendix Table I) ${ }^{1}$. For each paper in the seed set, we used Google Scholar to identify papers that cited it. Wohlin [19] recommends Google Scholar to avoid bias in favor of any specific publisher. We filtered the results given the publication date, including only those published from 2019 and later (i.e., we applied Inclusion criteria \#1). For the resulting papers, we screened them and applied Inclusion criteria \#2, \#3, and \#4. Finally, we analyzed the resulting papers' title and abstract in light of Inclusion criteria \#5.

On the first iteration, for the 15 papers in the seed set, we found 13 other papers, from which only six were considered relevant. The remaining seven were discarded for the following reasons: being book chapters or workshop papers, did not present ASD Teamwork factors, or were related to agile transition only. Given this, our data source for constructing the thematic network contained 21 papers.

\section{B. Thematic Network Construction}

To build the thematic network, we applied the thematic analysis process proposed by Cruzes and Dyba [18]. Their guideline is comprised of five steps: (i) data extraction; (ii) code data; (iii) translate codes into themes; (iv) create a model of higher-order themes; and (v) assess the trustworthiness of

\footnotetext{
${ }^{1}$ https://doi.org/10.6084/m9.figshare.14214431.v1
} 
the synthesis. All steps in this process were performed by the first author and checked by the other authors.

For the data extraction step, step (i), we extracted the Teamwork factors from the papers in our study's data source (see Section [14]), alongside text segments describing or explaining them. The output was a list of 74 ASD Teamwork factors that served as input for the next step. In step (ii), we coded the 74 ASD Teamwork factors' text segments identified in step (i) given their naming or description. For example, we labeled the factors Adaptability, Learning, Learning, and Team Adaptation into the code Learning in our further steps; while the factors Team Leadership, Shared Leadership, Leadership and Shared Responsibility were labelled with the code Shared Leadership.

Then, in step (iii), we translated the codes into themes. This step has some similarities with the previous one, given the mechanism that we adopted: group one or more codes into a theme representing them all. The themes comprised their representation into a specific Teamwork area that the authors judged valid given the codes' and factors' description. We grouped the codes that semantically represented a given theme and considered the ones that we judged essential to the theme (i.e., comprised essential attributes for the theme concept). For instance, we translated the codes Coordination, Performance Monitoring, Task Novelty, and Familiarity into Coordination. In this case, we considered that Coordination and Performance Monitoring have similar definitions (i.e., semantics) with regards to the higher-order theme (i.e., Coordination). For Task Novelty, according to Marsicano et al. [20], when its value is low, it is more likely that team members assign work between them adequately. This concept is also related to the concept of Familiarity, and according to the previous description of Task Novelty, we interpreted that they were also related to Coordination (i.e., task assignment).

After identifying 13 themes, we found that describing the ASD Teamwork in light of them was complex. Thus, we executed step (iv) by refining them into higher-order themes. We started by relating the 13 themes into a higher-order theme for the overall ASD Teamwork, resulting in a tree scheme, with $A S D$ Teamwork theme as the root node and the remaining 13 themes as leaf nodes.

Then, we identified new middle-level themes by grouping the leaf themes given their names and related factors. For example, we considered Cohesion a higher-order theme that comprises both Communication and Personality. The reasoning behind this is because Cohesion, as described in its related codes and factors, is directly related to the interpersonal attraction of team members and their willingness to continue working together. Based on this, we considered that the exchange of information between team members (i.e., Communication) and the mixture of personalities (i.e., Personality) contribute to it.

Notice that our goal was not to define a cause-consequence model, considering, for instance, the temporal relationship between the themes and codes, but to simplify the comprehension of ASD Teamwork dimensions. For example, even though Communication could be related to other themes, we associated it with the theme with the closest definition.

To perform step (v) and validate the synthesis's trustworthiness, we reviewed the process we adopted regarding reducing the bias of the researchers by relying on the description of codes and factors that comprise the themes based on the peer-review adopted. Besides, we believe that the themes are consistent and understandable.

\section{REsults}

This section presents the identified ASD Teamwork factors (see Table I) and the resulting thematic network (see Figure 1). In Table I, each line presents information for an identified code (i.e., ASD Teamwork factor). For each code, it presents the relative (i.e., "\%" column) and absolute frequency (i.e., "Freq" column) of the code's appearance on the seed set. Further, for improving understandability, column "Distribution" presents a visual representation of the code's frequency of appearance. Finally, column "Themes" displays the theme mapped for each code. The complete information on the thematic network process is available in the Appendix.

From this point forward, we explain the reasoning behind the relations that we have defined for the themes presented in Figure 1. The resulting thematic network is composed of four higher-level themes: Cohesion, Orientation, Shared Leadership, and Autonomy. As previously discussed in Section III-B, the relationship between Cohesion with Communication and Personality follows from the reasoning that both concepts are part of Cohesion.

Orientation refers to the team members' belief in the team goals' importance over individual members' goals and their propensity to take others' behavior into account during group interaction. We considered as sub-themes for this theme: Feedback, Coordination, Collaboration, and Learning. By analyzing these themes' concepts, we noticed that having the capabilities to coordinate the work among team members (i.e., Coordination) in a collaborative environment (i.e., Collaboration) that leverages constant feedback (i.e., Feedback) between the team members seemed strongly related to keeping team goals a top priority (i.e., Orientation).

Learning refers to the ability to understand and recognize deviations and readjust accordingly. Moreover, according to Ringstad et al. [11], it is also related to the development of shared mental models. Hence, we judged these capabilities as fundamental to keeping a good team Learning. Expertise is directly related to Learning because it comprises concepts such as Collective Knowledge, Redundancy, Adequate Skills, and Team Experience with Work, which we judged to be characteristics that influence the teams' learning capabilities.

The third higher-level theme is Shared Leadership, which relates to the provisioning of direction, structure, and support for the team members, with the responsibility and decision authority for doing so being shared between the team members. We judged that having good Management Mechanisms, which includes planning, discussing, implementing the required changes, and evaluating the success and the taken 
TABLE I

Codes Frequencies AND THEIR RELATED THEMES

\begin{tabular}{|c|c|c|c|c|}
\hline Codes & $\%$ & Freq & Distribution & Themes \\
\hline Coordination & $23.8 \%$ & 5 & {$[\mathrm{XXXXX]}$} & \multirow{4}{*}{ Coordination } \\
\hline Performance Monitoring & $42.85 \%$ & 9 & {$[\mathrm{XXXXXXXXX]}$} & \\
\hline Task Novelty & $4.75 \%$ & 1 & {$[\mathrm{X}]$} & \\
\hline Familiarity & $4.75 \%$ & 1 & {$[\mathrm{X}]$} & \\
\hline Culture & $19 \%$ & 4 & {$[\mathrm{XXXX}]$} & \multirow{4}{*}{ Organization Culture } \\
\hline Structure & $4.75 \%$ & 1 & {$[\mathrm{X}]$} & \\
\hline Team Size & $9.5 \%$ & 2 & {$[\mathrm{XX}]$} & \\
\hline Organization Support & $4.75 \%$ & 1 & {$[\mathrm{X}]$} & \\
\hline Individual Differences & $4.75 \%$ & 1 & {$[\mathrm{X}]$} & \multirow{3}{*}{ Members Personality } \\
\hline Heterogeneity & $4.75 \%$ & 1 & {$[\mathrm{X}]$} & \\
\hline Personality & $14.3 \%$ & 3 & {$[\mathrm{XXX}]$} & \\
\hline Management & $19 \%$ & 4 & {$[\mathrm{XXXX}]$} & \multirow{7}{*}{ Management Mechanisms } \\
\hline Planning & $4.75 \%$ & 1 & {$[\mathrm{X}]$} & \\
\hline Discussion & $4.75 \%$ & 1 & {$[\mathrm{X}]$} & \\
\hline Implementation & $4.75 \%$ & 1 & {$[\mathrm{X}]$} & \\
\hline Evaluation & $4.75 \%$ & 1 & [X] & \\
\hline Information Radiators & $4.75 \%$ & 1 & {$[\mathrm{X}]$} & \\
\hline Decision Making & $4.75 \%$ & 1 & {$[\mathrm{X}]$} & \\
\hline Orientation & $33.33 \%$ & 7 & {$[\mathrm{XXXXXXX]}$} & \multirow{9}{*}{ Orientation } \\
\hline Value Diversity & $4.75 \%$ & 1 & {$[\mathrm{X}]$} & \\
\hline Goals & $9.5 \%$ & 2 & {$[\mathrm{XX}]$} & \\
\hline Roles & $9.5 \%$ & $\frac{\pi}{2}$ & {$[\mathrm{XX}]$} & \\
\hline Holistic Team Involvement & $4.75 \%$ & 1 & {$[\mathrm{X}]$} & \\
\hline Team Experience in the Organization & $4.75 \%$ & 1 & {$[\mathrm{X}]$} & \\
\hline Trust & $23.8 \%$ & 5 & {$[\mathrm{XXXXX]}$} & \\
\hline Motivation & $4.75 \%$ & 1 & {$[\mathrm{X}]$} & \\
\hline Norms & $9.5 \%$ & 2 & {$[\mathrm{XX}]$} & \\
\hline Tools knowledge & $9.5 \%$ & 2 & {$[\mathrm{XX}]$} & \multirow{5}{*}{ Expertise } \\
\hline Collective Knowledge & $19 \%$ & 4 & {$[\mathrm{XXXX}]$} & \\
\hline Adequate Skills & $4.75 \%$ & 1 & {$[\mathrm{X}]$} & \\
\hline Redundancy & $33.33 \%$ & 7 & {$[\mathrm{XXXXXXX]}$} & \\
\hline Team Experience with Work & $4.75 \%$ & 1 & {$[\mathrm{X}]$} & \\
\hline Interdependence & $4.75 \%$ & 1 & {$[\mathrm{X}]$} & \multirow{2}{*}{ Collaboration } \\
\hline Collaboration & $33.33 \%$ & 7 & {$[\mathrm{XXXXXXX]}$} & \\
\hline Shared Leadership & $38.1 \%$ & 8 & {$[\mathrm{XXXXXXXX]}$} & \multirow{2}{*}{ Shared Leadership } \\
\hline Formal Leadership & $4.75 \%$ & 1 & {$[\mathrm{X}]$} & \\
\hline Autonomy & $19 \%$ & 4 & {$[\mathrm{XXXX}]$} & \multirow{2}{*}{ Autonomy } \\
\hline Task Control & $4.75 \%$ & 1 & {$[\mathrm{X}]$} & \\
\hline Awareness & $4.75 \%$ & 1 & {$[\mathrm{X}]$} & \multirow{3}{*}{ Feedback } \\
\hline Acceptance & $4.75 \%$ & 1 & {$[\mathrm{X}]$} & \\
\hline Feedback & $14.3 \%$ & 3 & {$[\mathrm{XXX}]$} & \\
\hline Learning & $38.1 \%$ & 8 & {$[\mathrm{XXXXXXXX]}$} & Learning \\
\hline Communication & $42.85 \%$ & 9 & {$[\mathrm{XXXXXXXXX]}$} & Communication \\
\hline Cohesion & $14.3 \%$ & 3 & {$[\mathrm{XXX}]$} & Cohesion \\
\hline
\end{tabular}




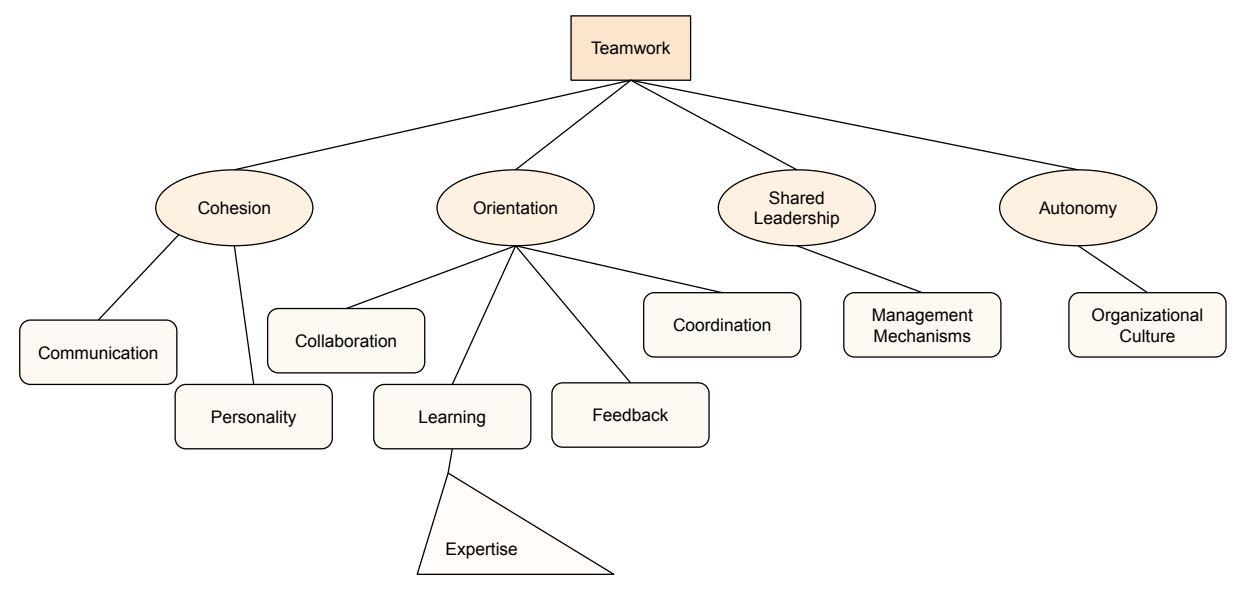

Fig. 1. ASD Teamwork Thematic Network

decisions, is essential for having working Shared Leadership. We decided to name this theme as Shared Leadership to adhere to agile teams' characteristic of being self-organized.

\section{DISCUSSION}

This section discusses this study's main findings and implications. Section IV presented this study's resulting thematic network, which helps organize the knowledge regarding ASD Teamwork factors. It eases knowledge sharing by defining the terminology to be used for the ASD Teamwork factors. For instance, we identified three terms for the factor Redundancy: Backup, Backup Behavior, and Redundancy.

Further, it provides a better understanding of the interrelationships between the factors (i.e., objects) of ASD Teamwork's knowledge field. However, notice that the proposed thematic network is not a cause-consequence model; instead, it comprehensively defines the themes (i.e., dimensions) that should be approached when describing ASD Teamwork. Moreover, the themes presented in Figure 1 respond our RQ defined in Section III.

It is intended to be used as a blueprint for both researchers and practitioners. However, it is not our intention to make the themes and their relations with ASD Teamwork something that must be followed as is, but to guide whoever intends to work towards their relevance based on literature findings.

The proposed thematic network also assists on identifying gaps in the ASD Teamwork knowledge field. Table I presents the distribution of occurrences for each of the identified ASD Teamwork factors. Notice that some factors have a high frequency, such as Performance Monitoring and Communication with nine appearances, while others only have one, such as Task Control and Awareness. Such results might indicate that the higher frequency factors are more important than ones with fewer, but it might only mean that they have been studied more. Thus, lines of research might follow from understanding the frequency distribution of the factors as shown in Table I.
Further, the proposed thematic network could be used to create a taxonomy for the ASD Teamwork field.

For researchers that intend to advance on the definition of ASD Teamwork constructs or its measurement, we expect them to consider all themes presented in our thematic network and complement or adapt their models/instruments based on the specific use-cases in which they fall. Taking previous studies into consideration when advancing the state-of-art is a premise. However, even the definition of another thematic network with the same purpose as the one defined in this study could bring valuable discussions around the topic that would benefit the academic community.

For practitioners, the thematic network can support their decision-making process. Practitioners can use it as a reference for understanding the factors and dimensions that comprise ASD Teamwork. With this, they can, for example, define mechanisms to monitor such dimensions and use the collected data as a reference to drive actions towards improving the team's performance. Furthermore, they can also extend our thematic network to achieve tailored instances to the team's context.

\section{THREATS TO VALIDITY}

This section discusses this study's threats to validity following the classification proposed by Wohlin et al. [21]: construct, internal, conclusion, and external validity.

- Construct validity: we analyzed the studies following a thematic analysis approach, in which multiple researchers participated to avoid researcher bias. However, we emphasize that removing researcher bias in qualitative research is virtually impossible. Thus, it is possible that the resulting thematic network (Figure 1) and codes (Table I) are not representative due to the researchers' interpretations. 
- Internal validity: to assure credibility in our findings, multiple researchers checked the extracted coding, themes, and the data presented in Figure 1.

- Conclusion validity: there is the risk that, since there is a threat to the construct validity of the thematic network, it influenced the extracted data and, consequently, our conclusions regarding the relationship between concepts.

- External validity: even though the data source was not a result of a broad literature review, we believe that it was representative of the state-of-the-art. Thus, we believe that the proposed thematic network is representative of agile TWQ in general. However, it might be possible that for specific situations, such as initial stages of agility adoption, including the transition from a traditional project management context, different factors could be included for ASD Teamwork.

\section{CONCLUSION}

This paper presented a thematic network that relates the main dimensions of ASD Teamwork. The thematic network consists of four higher-level themes: Cohesion, Orientation, Shared Leadership, and Autonomy. It also consists of additional nine themes.

It contributes to the field by defining a common terminology to be used for ASD Teamwork. It also provides a better understanding of the interrelationships between the factors of ASD Teamwork knowledge field. Thus, it advances the state-of-art in regards to ASD Teamwork construct definition. Further, it can support practitioners' decision-making process by being a reference for understanding the factors and dimensions of ASD Teamwork.

The main limitations of this study are related to the researchers' bias regarding the execution of the thematic network process explained in Section III-B; and the inclusion and exclusion criteria restrictiveness of the forward snowballing to build the data source for the thematic network construction. However, we still believe that the missing factors would still fall into the themes that we have defined.

The study identifies several opportunities for future work, including using the proposed thematic network to build a Teamwork taxonomy and as the basis for potentially refining the Teamwork model constructs (or instruments) presented in Freire et al. [14], Moe et al. [10], and Lindsjørn et al.[16].

\section{REFERENCES}

[1] R. Hoda, N. Salleh, and J. Grundy, "The rise and evolution of agile software development," IEEE Software, vol. 35, no. 5, pp. 58-63, 2018.

[2] L. Gren, A. Goldman, and C. Jacobsson, "Agile ways of working: A team maturity perspective,' Journal of Software: Evolution and Process, vol. 32, no. 6, p. e2244, 2020, e2244 smr.2244. [Online]. Available: https://onlinelibrary.wiley.com/doi/abs/10.1002/smr.2244

[3] K. Beck, M. Beedle, A. van Bennekum, A. Cockburn, W. Cunningham, M. Fowler, J. Grenning, J. Highsmith, A. Hunt, R. Jeffries, J. Kern, B. Marick, R. C. Martin, S. Mellor, K. Schwaber, J. Sutherland, and D. Thomas, "Manifesto for agile software development," http://www. agilemanifesto.org/, 2001.

[4] L. Gren and P. Lenberg, "Agility is responsiveness to change: An essential definition," in Proceedings of the Evaluation and Assessment in Software Engineering, 2020, pp. 348-353.
[5] A. Gomes, M. Neto, D. C. G. Valadares, M. Perkusich, D. Albuquerque, H. O. de Almeida, and A. Perkusich, "Evaluating the relationship of personality and teamwork quality in the context of agile software development," in SEKE, 2020.

[6] M. Hoegl and H. G. Gemuenden, "Teamwork quality and the success of innovative projects: A theoretical concept and empirical evidence," Organization science, vol. 12, no. 4, pp. 435-449, 2001.

[7] R. E. Kraut and L. A. Streeter, "Coordination in software development," Communications of the ACM, vol. 38, no. 3, pp. 69-81, Mar. 1995.

[8] T. Chow and D.-B. Cao, "A survey study of critical success factors in agile software projects," Journal of Systems and Software, vol. 81, no. 6, pp. 961-971, 2008.

[9] R. M. Fontana, I. M. Fontana, P. A. da Rosa Garbuio, S. Reinehr, and A. Malucelli, "Processes versus people: How should agile software development maturity be defined?" Journal of Systems and Software, vol. 97, pp. $140-155,2014$.

[10] N. B. Moe, T. Dingsøyr, and T. Dybå, "A teamwork model for understanding an agile team: A case study of a scrum project," Information and Software Technology, vol. 52, no. 5, pp. 480 - 491, 2010.

[11] M. A. Ringstad, T. Dingsøyr, and N. Brede Moe, Systems, Software and Service Process Improvement: 18th European Conference, EuroSPI 2011, Roskilde, Denmark, June 27-29, 2011. Proceedings. Berlin, Heidelberg: Springer Berlin Heidelberg, 2011, ch. Agile Process Improvement: Diagnosis and Planning to Improve Teamwork, pp. 167-178.

[12] L. Williams, K. Rubin, and M. Cohn, "Driving process improvement via comparative agility assessment," in Proceedings of the 2010 Agile Conference, ser. AGILE' 10. Washington, DC, USA: IEEE Computer Society, 2010, pp. 3-10.

[13] A. C. D. Batista, R. M. de Souza, F. Q. B. da Silva, L. de Almeida Melo, and G. Marsicano, "Teamwork quality and team success in software development: A non-exact replication study," in Proceedings of the 14th ACM / IEEE International Symposium on Empirical Software Engineering and Measurement (ESEM), ser. ESEM '20. New York, NY, USA: Association for Computing Machinery, 2020. [Online]. Available: https://doi.org/10.1145/3382494.3410632

[14] A. Freire, M. Perkusich, R. Saraiva, H. Almeida, and A. Perkusich, "A bayesian networks-based approach to assess and improve the teamwork quality of agile teams," Information and Software Technology, vol. 100, pp. 119-132, 2018. [Online]. Available: https://www.sciencedirect.com/ science/article/pii/S0950584917300204

[15] N. Moe, T. Dingsøyr, and E. Røyrvik, "Putting agile teamwork to the test - an preliminary instrument for empirically assessing and improving agile software development," in Agile Processes in Software Engineering and Extreme Programming, ser. Lecture Notes in Business Information Processing, P. Abrahamsson, M. Marchesi, and F. Maurer, Eds. Springer Berlin Heidelberg, 2009, vol. 31, pp. 114-123.

[16] Y. Lindsjørn, D. I. Sjøberg, T. Dingsøyr, G. R. Bergersen, and T. Dybå, "Teamwork quality and project success in software development: A survey of agile development teams," Journal of Systems and Software, vol. 122, pp. 274 - 286, 2016. [Online]. Available: http://www.sciencedirect.com/science/article/pii/S016412121630187X

[17] R. M. Lindsay and A. S. C. Ehrenberg, "The design of replicated studies," The American Statistician, vol. 47, no. 3, pp. 217-228, 1993. [Online]. Available: http://www.jstor.org/stable/2684982

[18] D. S. Cruzes and T. Dyba, "Recommended steps for thematic synthesis in software engineering," in 2011 International Symposium on Empirical Software Engineering and Measurement, 2011, pp. 275-284.

[19] C. Wohlin, "Guidelines for snowballing in systematic literature studies and a replication in software engineering," in Proceedings of the 18th International Conference on Evaluation and Assessment in Software Engineering - EASE '14, ser. EASE '14. New York, NY, USA: Association for Computing Machinery, 2014. [Online]. Available: https://doi.org/10.1145/2601248.2601268

[20] G. Marsicano, F. Q. da Silva, C. Seaman, and B. G. Adaid-Castro, "The teamwork process antecedents (tpa) questionnaire: developing and validating a comprehensive measure for assessing antecedents of teamwork process quality," Empirical Software Engineering, vol. 25, pp. 3928-3976, 2020.

[21] C. Wohlin, P. Runeson, M. Hst, M. C. Ohlsson, B. Regnell, and A. Wessln, Experimentation in Software Engineering. Springer Publishing Company, Incorporated, 2012. 